\title{
Hedonic Hot Spot in Nucleus Accumbens Shell: Where Do $\mu$-Opioids Cause Increased Hedonic Impact of Sweetness?
}

\author{
Susana Peciña and Kent C. Berridge \\ Department of Psychology, University of Michigan, Ann Arbor, Michigan 48109
}

\begin{abstract}
$\mu$-Opioid systems in the medial shell of the nucleus accumbens contribute to hedonic impact ("liking") for sweetness, food, and drug rewards. But does the entire medial shell generate reward hedonic impact? Or is there a specific localized site for opioid enhancement of hedonic "liking" in the medial shell? And how does enhanced taste hedonic impact relate to opioid-stimulated increases in food intake? Here, we used a functional mapping procedure based on microinjection Fos plumes to localize opioid substrates in the medial shell of the nucleus accumbens that cause enhanced "liking" reactions to sweet pleasure and that stimulate food intake. We mapped changes in affective orofacial reactions of "liking"/“disliking" elicited by sucrose or quinine tastes after D-Ala ${ }^{2}-\mathrm{N}-\mathrm{Me}-\mathrm{Phe}^{4}$-Glycol ${ }^{5}$-enkephalin (DAMGO) microinjections in rats and compared hedonic increases to food intake stimulated at the same sites. Our maps indicate that opioid-induced increases in sucrose hedonic impact are generated by a localized cubic millimeter site in a rostrodorsal region of the medial shell. In contrast, all regions of the medial shell generated DAMGO-induced robust increases in eating behavior and food intake. Thus, our results identify a locus for opioid amplification of hedonic impact and reveal a distinction between opioid mechanisms of food intake and hedonic impact. Opioid circuits for stimulating food intake are widely distributed, whereas hedonic "liking" circuits are more tightly localized in the rostromedial shell of the nucleus accumbens.
\end{abstract}

Key words: nucleus accumbens; medial shell; reward; pleasure; palatability; affect; hedonics; $\mu$ agonist; opioid; opiate; DAMGO; feeding; food intake; incentive motivation; incentive salience; shell; dopamine; mesolimbic; limbic; microinjection

\section{Introduction}

Opioid neurotransmission in the medial shell of the nucleus accumbens is thought to participate in generating hedonic impact for drug rewards and for natural sensory pleasures such as sweetness (Zhang et al., 1998; Peciña and Berridge, 2000; Kelley et al., 2002, 2004; Robbins and Everitt, 2002; Berridge, 2003; Koob, 2003; Bodnar et al., 2005; Kalivas and Volkow, 2005). Opioid shell circuits also contribute to eating behavior and other incentive motivation (Majeed et al., 1986; Mucha and Iversen, 1986; Evans and Vaccarino, 1990; Zhang et al., 1998). However, little is known about the precise location of hedonic opioid circuits within the nucleus accumbens or about the relationship between hedonic ("liking") versus motivational ("wanting") opioid functions.

Where in the accumbens shell does $\mu$-opioid activation cause increased sweet "liking" for a sweet taste? Changes caused by brain manipulations in the hedonic impact of sweetness in particular can be measured relatively specifically, even in animals, via behavioral taste reactivity patterns, which are affective orofacial expressions that are homologous in human infants, apes, mon-

Received June 7, 2005; revised 0ct. 7, 2005; accepted 0ct. 24, 2005.

This work was supported by National Institutes of Health Grants DA015188 and MH63649. We are grateful to Prof. Sarah W. Newman for helpful guidance on neuroanatomical issues and to Phillip Hoberg and Kyle Smith for assistance with Fos plume mapping.

Correspondence should be addressed to Susana Peciña, Department of Psychology, University of Michigan, Ann Arbor, Ml 48109-1109. E-mail: pesu@umich.edu.

DOI:10.1523/JNEUROSCI.2329-05.2005

Copyright $\odot 2005$ Society for Neuroscience $\quad$ 0270-6474/05/2511777-10\$15.00/0 keys, and rats (Berridge, 2000). Sweet tastes elicit positive "liking" expressions (tongue protrusions, etc.), whereas bitter tastes instead elicit facial "disliking" reactions (gapes, etc.). Positive "liking" reactions to sucrose tastes are increased in rats by morphine microinjection into the nucleus accumbens medial shell (Peciña and Berridge, 2000), as well as by intraventricular or systemic administration, whereas aversive "disliking" reactions to bitter are suppressed (Parker et al., 1992; Doyle et al., 1993; Peciña and Berridge, 1995; Rideout and Parker, 1996).

The ability of opioid $\mu$ agonists to increase food intake appears to be anatomically distributed widely throughout the entire nucleus accumbens shell and related structures (Evans and Vaccarino, 1990; Zhang et al., 1998; Peciña and Berridge, 2000; Echo et al., 2002; Levine et al., 2004; Will et al., 2004). Conversely, $\mu$-opioid antagonists at diverse sites in the nucleus accumbens suppress eating and intake, especially for palatable foods (Cooper and Higgs, 1994; Bodnar et al., 1995; Kelley et al., 1996; Levine and Billington, 1997).

Is opioid generation of hedonic impact equally widely distributed in the accumbens shell? Or are opioid mechanisms that cause increased "liking" reactions to sensory pleasure more localized than generation of food intake? Here, we used taste reactivity and a microinjection Fos plume technique to map enhancement of hedonic impact of sweetness caused by D-Ala ${ }^{2}-\mathrm{N}-\mathrm{Me}-\mathrm{Phe}^{4}$ Glycol $^{5}$-enkephalin (DAMGO) in the medial accumbens shell, comparing "liking" to food intake effects for the same sites in the same rats. Our findings indicate that opioid-generated "liking" mechanisms are restricted to a single cubic millimeter site local- 
ized in the rostrodorsal quarter of the medial shell of the nucleus accumbens. Outside this "opioid hedonic hot spot," DAMGO microinjections left sucrose "liking" reactions unchanged or even suppressed, despite stimulating increased food intake at virtually all sites throughout the entire medial shell. These results identify a locus for $\mu$-opioid substrates that generate hedonic impact in the nucleus accumbens medial shell and indicate that $\mu$-opioid circuits in the medial shell can stimulate food intake via either hedonic or nonhedonic mechanisms, depending on the precise location.

\section{Materials and Methods \\ General design}

Our Fos plume analysis measures the spread of Fos-like immunoreactivity (FLI) around local microinjection sites caused by DAMGO; elevated Fos expression is detected by comparison to normal tissue and to vehicle control microinjections (Peciña and Berridge, 2000; Smith and Berridge, 2005). The intensity of drug-evoked behavioral effects is plotted by colorcoded, plume-sized symbols onto sagittal maps of the medial shell of the nucleus accumbens (Paxinos and Watson, 1996). Sagittal maps are used to recognize regional differences because they allow the entire medial shell to be viewed at a glance. Fos plume-based maps for opioid generation of food intake, positive reward hedonic "liking" functions, and negative aversive "disliking" functions were constructed separately. Separate maps were also constructed for two doses of DAMGO $(0.01$ and $0.1 \mu \mathrm{g})$ compared with vehicle effects at the same sites $(0 \mu \mathrm{g})$. To directly compare "liking" and eating DAMGO effects at identical sites in the same rats, a within-subject design was used to test each rat for both affective taste reactivity patterns and eating behavior. The order of test type and of vehicle/drug dose was counterbalanced across rats.

\section{Subjects}

A total of 50 male Sprague Dawley rats born at the University of Michigan (270-320 g at the time of surgery) were used for behavioral shell mapping experiments and were housed in pairs $\left(\sim 21^{\circ} \mathrm{C} ; 12 \mathrm{~h}\right.$ light/dark cycle) with food (Purina Rat Chow; Purina Mills, St. Louis, MO) and water (tap water) available ad libitum. Animals were handled extensively before surgery and testing to habituate them to handling procedures. All studies were approved by the University Committee on Use and Care of Animals of the University of Michigan in compliance with National Institutes of Health standards.

\section{Surgery}

Rats were pretreated with $0.1 \mathrm{ml}$ of atropine sulfate, anesthetized with a mixture of ketamine $\mathrm{HCl}$ and xylazine ( 80 and $5 \mathrm{mg} / \mathrm{kg}$, respectively), and stereotaxically implanted with bilateral microinjection cannulas (23 gauge; stainless steel). The incisor bar was set at $5.0 \mathrm{~mm}$ above interaural zero to slant the skull upward and avoid penetrating the lateral ventricles. Each rat was assigned individual coordinates from a group set that ranged to essentially fill the entire medial shell of nucleus accumbens. Cannula placements were staggered across 44 rats at spatial intervals of $\sim 0.35 \mathrm{~mm}$ within the following dimensions: anteroposterior (AP), 3.4-1.9 mm anterior to bregma (corresponding to AP, 2.2-0.7 mm with a flat skull) and dorsoventral (DV), -7.5 to $-9.8 \mathrm{~mm}$ from skull (corresponding to DV, -6.2 to $-8.4 \mathrm{~mm}$ with a flat skull); mediolateral (ML) was always \pm $0.9 \mathrm{~mm}$ (Paxinos and Watson, 1996). To serve as anatomical controls for diffusion, six additional rats were implanted in sites outside the nucleus accumbens, in structures dorsal (lateral septum) or ventral (medial olfactory tubercle) to the medial shell. Microinjection guide cannulas were anchored to the skull with screws and acrylic cement. A stainless-steel obturator was inserted into each guide cannula to prevent occlusions.

All rats were simultaneously implanted with chronic bilateral oral cannulas for infusion of taste solutions into the mouth. A 19 gauge needle connected to cannula tubing was inserted adjacent to the first auxiliary molar and routed within the zygomatic arch to exit at the scalp incision. The oral cannulas [heat-flared polyethylene (PE)-100 tubing] were anchored inside the mouth by Teflon washers, fitted with 19 gauge steel cannulas at the skull, and cemented to skull screws along with the accumbens microinjection cannulas. Rats received postoperative penicillin (aquacillin; 45,000 U, i.m.) and were allowed to recover from surgery at last 1 week before behavioral test sessions began.

\section{Drugs and microinjections}

To avoid accumulation of excessive local damage caused by too many microinjections, the number of microinjections received by any rat was limited to six. DAMGO (Sigma, St. Louis, MO) was dissolved in sterile $0.9 \%$ saline, and DAMGO doses were selected to be within the lowest range that reliably elicits increased eating after accumbens microinjections: 0.0 (vehicle), 0.01 , and $0.1 \mu \mathrm{g}$ in a $0.2 \mu \mathrm{l}$ volume, based on pilot studies (our unpublished observations) and on previous reports (Zhang and Kelley, 1997; Zhang et al., 1998). Low doses were preferred here to minimize diffusion from microinjection sites that could obscure mapping of functional localization. Microinjections were always given bilaterally (doses refer to the amount given per unilateral side; therefore, doses per rat were double these amounts). The testing order of drug doses and vehicle was counterbalanced between rats. Only one test was given per day, and an at least $48 \mathrm{~h}$ rest period was allowed between successive tests.

To give a microinjection, a rat was gently hand-held or cradled in the experimenter's arm, while the stylets were removed and one cannula at a time was attached to a syringe pump via PE-20 tubing. A $0.2 \mu$ l volume of drug or vehicle was injected at a rate of $0.3 \mu \mathrm{l} / \mathrm{min}$ using a stainless-steel injector cannula (29 gauge), which extended $2.0 \mathrm{~mm}$ beyond the ventral tip of the cannula guide. The injector was kept in place for $1 \mathrm{~min}$ after the microinjection before the obturators were replaced.

\section{Experiment 1: accumbens DAMGO modulation of affective reactions elicited by sucrose or quinine}

Measuring of hedonic impact: the taste reactivity paradigm. The taste reactivity paradigm (Grill and Norgren, 1978) provides a way of specifically assessing the hedonic impact of a sucrose reward by measuring affective reactions elicited by oral infusions of taste stimuli that are homologous in human infants, apes, monkeys, and rats (Grill and Berridge, 1985; Berridge, 1996, 2000). Positive hedonic reactions (rhythmic and lateral tongue protrusions, etc.; "liking" patterns) are normally elicited by sucrose taste. Negative aversive reaction (gapes, headshakes, etc.; "disliking" patterns) are normally elicited by quinine taste. Positive "liking" reactions of rats are altered by many of the same manipulations that increase human subjective ratings of taste palatability, such as physiological hunger/satiety, psychological conditioned preferences/aversions, and neural manipulations, indicating that these behavioral "liking" reactions reflect a basic evaluation of the hedonic impact or palatability of a taste (Berridge, 2000).

Taste reactivity testing. Rats were habituated to the testing environment for $3 \mathrm{~d}$ before the beginning of testing and to the taste reactivity procedure with an oral infusion of distilled water for 1 additional day. Taste reactivity tests began $\sim 15$ min after the last microinjection [based on previous studies and pilot observations of rapid onset for hedonic enhancement by shell opioid microinjections (Peciña and Berridge, 2000)]. For the taste reactivity test, a PE-10 nozzle attached to a stimulus delivery line (PE-50 tubing) was inserted into the rat's oral cannula immediately after bilateral microinjections, and the rat was placed into a transparent test chamber for $15 \mathrm{~min}$ habituation. A $1 \mathrm{ml}$ solution of $0.1 \mathrm{M}$ sucrose was infused into the rat's mouth by syringe pump for $1 \mathrm{~min}$ at a rate of $1 \mathrm{ml} / \mathrm{min}$ beginning at $15 \mathrm{~min}$ after microinjection. A mirror positioned beneath the transparent floor reflected a view of the rat's face and mouth into the close-up lens of a video camera to permit videotaping of affective facial and body reactions. Sucrose at a $0.1 \mathrm{M}$ concentration normally elicits moderate numbers of positive "liking" reactions (and no aversive reactions), and increases in positive hedonic reactions produced by DAMGO can be observed above this moderate level. A 1 min water rinse followed immediately. Ten minutes later, $1 \mathrm{ml}$ of $3 \times 10^{-4}$ quinine $\mathrm{HCl}$ solution was infused to elicit aversive "disliking" reactions beginning at $25 \mathrm{~min}$ after microinjection (rate, $1 \mathrm{ml} / \mathrm{min}$; duration, $1 \mathrm{~min}$ ). Sucrose was always the first stimulus of the day, because the primary focus of our study was opioid modulation of positive hedonic impact, and it was imperative that sucrose "liking" reactions be pure in the sense of being uninfluenced by any previous stimulus. Fortunately, aversive 
"disliking" reactions to bitter quinine are generally robust at $3 \times 10^{-4} \mathrm{M}$ concentration, so quinine could safely be given as a second stimulus, especially because the $10 \mathrm{~min}$ interval between sucrose and quinine infusions allowed rest and dissipation of sucrose effects before quinine testing, and the constant stimulus order meant that any remaining order effects for quinine were equated across vehicle and DAMGO tests.

Taste reactivity scoring. Behavioral analysis of positive and negative affective reactions was done in slow motion (1/10 of actual speed) (Movie 1 , available at www.jneurosci.org as supplemental material) by an observer blind to drug treatment condition following procedures described previously (Berridge, 2000). Positive hedonic ("liking") reactions included lateral tongue protrusions (large protrusions of the tongue extending away for the midline), midline tongue protrusions (smaller rhythmic midline protrusions), and paw licking. Negative aversive ("disliking") reactions included gapes (large opening of the mouth with a retraction of the lower lip), forelimb flails (rapid waving of one or both forelimbs), head shakes, face wiping, and chin rubs (pushing chin against the side or floor of the test chamber). Neutral reactions (less strongly linked to hedonic/aversive evaluations) were considered to be rhythmic mouth movements and passive drip of the solution. Lateral tongue protrusion, gapes, forelimb flails, head shakes, and chin rubs were counted as discrete single actions each time they occurred. Continuous or repetitive actions were counted in time bins: midline tongue protrusions ( $2 \mathrm{~s}$ bins), paw licking ( $5 \mathrm{~s}$ bins), and face wiping ( $5 \mathrm{~s}$ bins). These procedures allow balanced representation of all reaction types when they are summed together into a total positive hedonic "liking" score versus a total aversive "disliking” score (Berridge, 2000).

\section{Experiment 2: increased food intake after DAMGO in the medial shell}

Behavioral eating test. The effects of DAMGO on eating behavior and food intake were assessed in a voluntary feeding test. Food intake and taste reactivity tests were counterbalanced in order and spaced at least $2 \mathrm{~d}$ apart.

Immediately after a microinjection, rats were placed in transparent chambers $(23 \times 20 \times 45 \mathrm{~cm} ; 1 \mathrm{~cm}$ corncob bedding $)$ that contained a dish of preweighed food ( $\sim 20 \mathrm{~g}$; Purina Rat Chow) and were left undisturbed during the $60 \mathrm{~min}$ intake test session. A water spout was also provided. Behavior of each rat was videotaped. At the end of the session, the amount of food left was weighed and recorded. Bedding was inspected for food crumbs. Videotapes were scored minute-by-minute by an observer blind to microinjection testing conditions for duration of eating behavior, drinking behavior, and several other behaviors (grooming, treading, locomotion, etc.).

\section{Histology}

After the completion of testing, each brain was prepared for two different analyses: (1) local Fos plume densities and diameters were measured after microinjections of DAMGO doses or vehicle in rats not used for behavioral tests; (2) microinjection centers were mapped by ink microinjection and cresyl violet staining in rats used for behavioral testing and used to plot site centers in functional maps.

\section{Fos-like protein immunohistochemistry}

Each rat was microinjected with DAMGO or vehicle $(0.2 \mu \mathrm{l})$ and $1 \mathrm{~h}$ later was deeply anesthetized with sodium pentobarbital. Perfusions were performed $75 \mathrm{~min}$ after drug microinjection, because the translation of c-fos mRNA to Fos protein has been found to reach peak levels between 60 and 120 min (Muller et al., 1984). Rats were perfused intracardially with 250 $\mathrm{ml}$ of $0.2 \mathrm{M}$ sodium phosphate buffered saline containing $0.1 \%$ sodium nitrite, followed by $250 \mathrm{ml}$ of $4 \%$ paraformaldehyde in $0.1 \mathrm{M}$ sodium phosphate buffer (NaPB). Brains were removed and placed in $4 \%$ formaldehyde for $2 \mathrm{~h}$ and then placed in $30 \%$ sucrose at least overnight, then sectioned at placed in $0.2 \mathrm{~m} \mathrm{NaPB}, \mathrm{pH}$ 7.4. To visualize Fos-likeimmunoreactive neurons, we used the avidin-biotin procedure. Brain sections were first immersed in blocking solution [3\% normal goat serum (NGS) and $0.3 \%$ Triton X-100 in Tris PBS (TPBS)] for $1 \mathrm{~h}$ at room temperature and then incubated for $24 \mathrm{~h}$ at room temperature with a rabbit polyclonal antiserum directed against the $\mathrm{N}$-terminal region of the Fos protein (Sigma) at a dilution of 1:5000 in TPBS, 1\% NGS, and $0.3 \%$
Triton X-100. To reduce background staining, the antiserum was preabsorbed with acetone-dried rat liver powder overnight at $4^{\circ} \mathrm{C}$. After primary antibody incubation, the tissue was exposed to a goat anti-rabbit, biotinylated secondary IgG (Santa Cruz Biochemicals, Santa Cruz, CA) diluted 1:200 and then to avidin-biotin-peroxidase complex for $1 \mathrm{~h}$ at room temperature. A nickel diaminobenzidine glucose oxidase reaction was used to visualize black-brown Fos-like-immunoreactive nuclei. Finally, sections were washed in Tris buffer, mounted onto slides from tap water, air-dried, dehydrated in a graded series of alcohols, cleared in xylene, and coverslipped. Fos-like immunoreactivity was visualized using a Leica (Nussloch, Germany) microscope coupled to a Spot RT slider (Diagnostic Instruments, Sterling Heights, MI) using Spot software (version 3.3).

\section{Fos plume mapping}

Our procedure for mapping opioid-induced Fos plumes immediately surrounding a local microinjection site was modified slightly from previous studies (Peciña and Berridge, 2000; Smith and Berridge, 2005). Briefly, Fos-labeled cells on tissue surface with $5-40 \times$ magnification were counted individually within blocks $(125 \times 125 \mu \mathrm{m})$ at point locations spaced at $125 \mu \mathrm{m}$ intervals along each of seven radial arms emanating from the center of the microinjection site $(45,90,135,180,225,270$, $315^{\circ}$ ) (see Fig. 1). The magnitude of local Fos elevations was plotted as Fos plume zones surrounding the microinjection sites.

DAMGO Fos plumes reflect increases in local Fos-like protein expression over control levels caused by $\mu$-opioid agonist. To assess that, a three-way comparison was made of (1) normal baseline Fos expression in medial shell of nucleus accumbens tissue in brains without microinjection cannulas, (2) control plumes surrounding vehicle microinjections (i.e., FLI induced by cannula track damage, or vehicle pressure wave or chemical composition), and (3) elevated DAMGO plumes surrounding the site of drug microinjections (see Fig. 1).

Fos plumes surrounding DAMGO microinjections were mapped as zones of intense or moderate elevation of Fos expression, each identified by two criteria (see Fig. 1): (1) absolute increases over normal tissue levels of FLI $[10 \times$ (intense) or $5 \times$ (moderate) elevation over normal tissue counts sampled in the absence of any cannula track]; (2) vehicle-relative increases over FLI surrounding control microinjections ( 5 or $2 \times$ elevation at the same point locations over vehicle microinjection-induced levels).

\section{Mapping procedure for microinjection behavioral effects}

Functional maps of elicited eating and "liking" were constructed using a mapping procedure to depict the behavioral consequences of DAMGO at each microinjection site and to make quantitative comparisons across sites and across eating versus "liking" effects for the same site. Cannula placements were first located by plotting the site center in the coronal plane, because the coronal plane has been mapped with higher resolution (i.e., more slices/maps per millimeter of brain) than sagittal or horizontal planes (Paxinos and Watson, 1996). Using the coronal plane that has the most sections per millimeter gives the most accurate initial mapping of the three-dimensional (3-D) coordinates of a site (the other two dimensions are continuous in the atlas page). If the anteroposterior plane of the site was judged to fall between two coronal atlas pages, its AP bregma coordinate was assigned to be intermediate to their levels. The three coordinates obtained for each site center (AP, DV, ML) were then replotted into a site location in a sagittal map of medial shell (ML, \pm 0.9$)$. A sagittal plane was chosen for reconstructing all final Fos plume maps of function localization, because it allows the entire medial shell of nucleus accumbens to be viewed at once in a single map, which facilitates localization of function.

Separate maps were constructed for 0.01 and $0.1 \mu \mathrm{g}$ doses of DAMGO and for each type of behavioral effect (hedonic sucrose "liking," aversive quinine "disliking," spontaneous eating, and food intake). Symbols were assigned to each microinjection site, and the sizes of symbols reflected the mean size of Fos plumes produced by DAMGO at that dose (separately representing intense and moderate FLI zones).

Colors were assigned to each symbol based on the functional magnitude of behavioral effect produced by a DAMGO dose at that site com- 
pared with vehicle at the same site (e.g., hedonic "liking" reaction increase, aversive "disliking" reaction decrease, food intake increase). DAMGO effects on "liking" reactions to sucrose were mapped by colors reflecting the percentage change from vehicle microinjection at the same site: enhancement greater than $+250,+200,+150$, and +125 ; no change (less than $\pm 125 \%$ change from vehicle); and suppression greater than -125 and $-150 \%$. DAMGO effects on aversive reaction were mapped as pure suppressions: decreases less than $-75 \%$ ( $<25 \%$ of vehicle), less than $-50 \%$, less than $-25 \%$ ( $<75 \%$ of vehicle), and no change $(<125 \%$ change). Eating effects were mapped as percentage increase in eating duration from vehicle (10 s was added to every score as a constant to prevent near-zero vehicle scores from creating excessive DAMGO percentage increases): increases greater than $+800,+400,+200$, and +150 and no change $(<125 \%$ baseline $)$.

To further analyze localization of function, the sagittal map of the medial shell was subdivided into a 36-square grid (six columns $\times$ six rows). This allowed us to quantify intensity gradients of behavioral effects along rostrocaudal and dorsoventral axes.

\section{Statistical analysis}

The effects of DAMGO (drug) were first assessed using a two-way ANOVA (drug-by-site), which compared effects of different doses at different sites on taste reactivity, food intake, and eating duration data. Post hoc Tukey's multiple comparison tests were used when a factor was significant to make subsequent specific paired comparisons.

\section{Results}

Fos plumes: identifying zones of local neuronal activation

All microinjection studies generally state where a drug has been placed, but to localize function, it is also helpful to know how far the drug has acted. Fos plumes are a useful way to gain quantitative information about the functional size of activation spheres produced by drug microinjection, at least for drugs such as DAMGO that induce local Fos transcription. Local Fos plumes revealed the spread and size of functional modulation caused by $\mu$-opioid activation, expressed as elevated Fos around the site of DAMGO microinjections.

It is not yet known whether local Fos expression reflects direct opioid action on receptors of the same neuron that shows FLI, or else indirect FLI in a neighbor induced via local circuits from adjacent neurons that contain opioid receptors. However, in either case, the Fos plume reflects a sphere of local functional modulation induced by the drug, and its parameters provide objective information on sphere intensity and size, indicating the spread of neuronal activation. Most important for function localization, the boundaries of the plume reveal where the drug stops having intense functional impact (even if drug molecules physically spread further in lower concentrations insufficient to trigger gene transcription). Thus, a Fos plume helps reveal the size of the zone most likely responsible for functional effects of the drug microinjection.

For each Fos plume, we discriminated between zones of intense versus moderate Fos expression elevation. An inner zone of intense Fos elevation was defined as the mean radius within which (1) absolute FLI increase over normal tissue levels was increased by at least 1 order of magnitude, and (2) relative FLI over equivalent locations after control microinjections was at least doubled over vehicle microinjection levels. An outer zone of moderate Fos elevation was defined as the mean radius within which (1) absolute Fos expression over normal tissue was increased by $5 \times$ (but not $10 \times$ ), and (2) relative Fos expression over vehicle was increased by $5 \times$. [Note: the slight elevation over normal tissue FLI in inner zone baseline caused by vehicle microinjections (e.g., pressure wave, vehicle chemical stimulation, or local damage) imposed a ceiling on vehicle-relative increases in
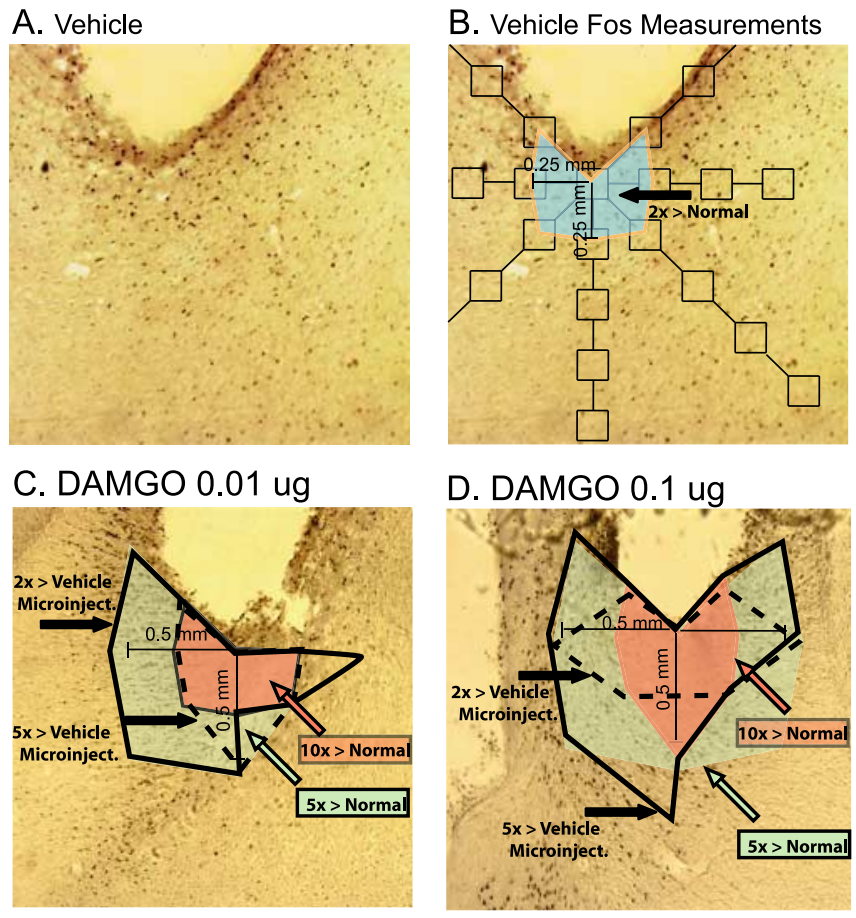

Figure 1. Fos plumes. $\boldsymbol{A}$, Coronal section showing point sample positions used to identify local Fos plumes around microinjection site $(125 \times 125 \mu \mathrm{m}$ blocks on radial arms extending from center; $5 \times$ magnification) after vehicle microinjection. $\boldsymbol{B}$, Coronal section showing Fos expression after vehicle microinjection. $\boldsymbol{C}$, Photograph map shows a representative Fos plume after a microinjection of low-dose $(0.01 \mu \mathrm{g})$ DAMGO (in $0.2 \mu$ l volume). D, Fos plume produced by higher-dose $(0.1 \mu \mathrm{g} / 0.2 \mu \mathrm{l})$ DAMG0. Intense elevation in Fos expression compared with normal levels was compared with normal accumbens shell tissue (absolute increases; $10 \times$ denoted by red color; $5 \times$, green; $2 \times$, blue) and over vehicle microinjection levels at equivalent points surrounding a cannula site (relative increases; $2 \times$, by thick dotted line; $5 \times$, thin dotted line; top right). Microinject., Microinjection.

drug-induced inner Fos expression limited to under five times vehicle levels (even if DAMGO caused a $10 \times$ absolute increase over normal tissue in the same inner zone) (Smith and Berridge, 2005).]

The size of Fos plume caused by each $0.2 \mu$ l volume microinjection depended on DAMGO dose [0 (vehicle), $0.01 \mu \mathrm{g}, 0.1 \mu \mathrm{g}$ ] (Fig. 1). Microinjections of a low dose $(0.01 \mu \mathrm{g})$ of DAMGO caused an intense inner Fos plume of $0.18 \pm 0.08 \mathrm{~mm}$ in radius and a moderate outer zone of additional $0.22 \pm 0.04 \mathrm{~mm}$ in radius. Fos plumes typically had an arrowhead shape (Fig. 1). To estimate plume volume, however, this seemed close enough to circular to assume an approximately spherical shape, and we calculated the inner intense zone after $0.01 \mu \mathrm{g}$ of DAMGO to be a sphere, $\sim 0.025 \mathrm{~mm}^{3}$ in volume (absolute $10 \times$ increase over normal; relative $2 \times$ increase over vehicle), with the outer sphere of $\sim 0.27 \mathrm{~mm}^{3}$ in volume. By comparison, the higher $0.1 \mu \mathrm{g}$ dose of DAMGO caused an inner intense zone of $0.21 \pm 0.07$ in radius, and so a 3-D inner sphere of $\sim 0.039 \mathrm{~mm}^{3}$ in volume. This $0.1 \mu \mathrm{g}$ dose produced an outer moderate Fos plume zone of an additional $0.29 \pm 0.04$ in radius, and so therefore an outer sphere of $\sim 0.52 \mathrm{~mm}^{3}$ in total volume. To provide perspective on the extent to which these spheres fill the larger structure, we estimated the entire medial shell to be $\sim 2.87 \mathrm{~mm}^{3}$ in total volume. Therefore, the inner zone of a low dose $0.01 \mu \mathrm{g}$ plume filled $\sim 1 \%$ of total shell volume and its outer zone filled $10 \%$, whereas a higher $0.1 \mu \mathrm{g}$ dose inner plume zone filled $\sim 1.5 \%$ and its outer plume filled $12 \%$ of total medial shell volume. Of course, for microinjections near borders with other structures, some plume spheres may have straddled 

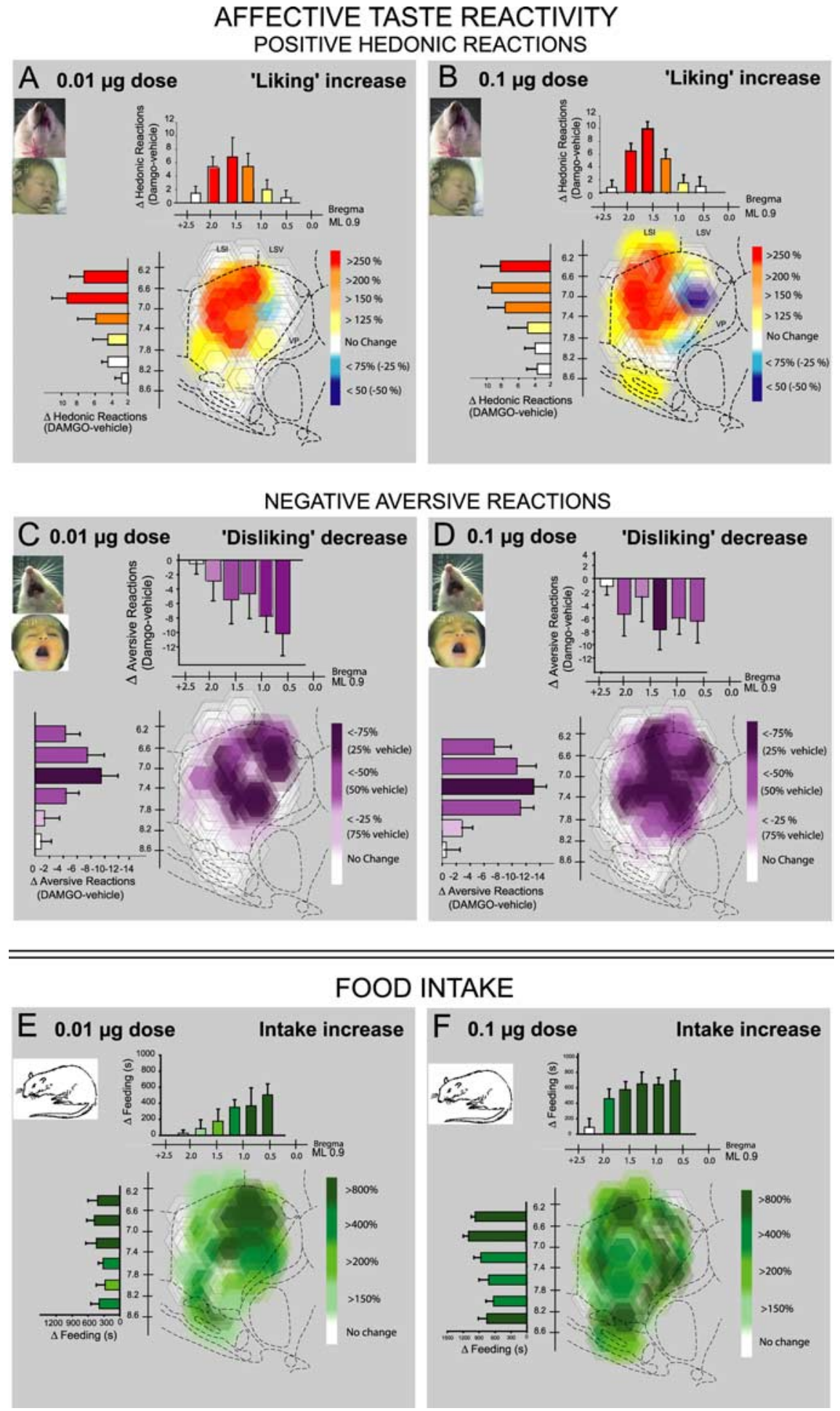

Figure 2. Changes in hedonic "liking" $(\boldsymbol{A}, \boldsymbol{B})$, aversive "disliking" $(\boldsymbol{C}, \boldsymbol{D})$, and food intake $(\boldsymbol{E}, \boldsymbol{F})$ caused by DAMG0 microinjections. Doses are shown separately: $\boldsymbol{A}, \boldsymbol{C}, \boldsymbol{E}$, Low dose $(0.01 \mu \mathrm{g} / 0.2 \mu \mathrm{l}) ; \boldsymbol{B}, \boldsymbol{D}, \boldsymbol{F}$, high dose $(0.01 \mu \mathrm{g} / 0.2 \mu \mathrm{l})$. Behavioral changes are expressed as within-subject percentage changes from vehicle microinjections at the same sites (vehicle, $100 \%$ ). Bilateral accumbens sites from left and right brains of each rat are collapsed together here into a unilateral single map of accumbens for simplicity. $A, B$, Enhancement of positive hedonic impact for sucrose taste. The colors denote intensity of "liking" change from vehicle levels, and symbol size shows the diameter of intense Fos plumes ( $10 \times$ elevation above normal, surrounded by semitransparent halos that show diameter of moderate Fos plumes). The bar graphs show absolute magnitudes of behavioral change caused by microinjections in each AP column or DV row (DAMGO minus vehicle). The colors in bars denote percentage behavioral change, dividing DAMGO by vehicle baseline as in maps. The highest increase in positive hedonic reactions was evoked by DAMG0 microinjection in the rostrodorsal quarter of medial shell, represented in orange and red in the diagram. Decreases in positive affective reactions are coded in blue (with diagonal black lines). $C, D$, Suppression of negative aversive impact of quinine taste. Intensity of suppression of "disliking" reactions are shown by shades of purple; otherwise, as above. $\boldsymbol{E}$, F, Stimulation of food intake. Increases of eating (cumulative duration of eating behavior) are shown by shades of green; otherwise, as above. All sagittal atlas maps at ML \pm 0.9 are from Paxinos and Watson (1996). See also supplemental material (available at www.jneurosci.org; see both (though never penetrating more than $\sim 0.5 \mathrm{~mm}$ into adjacent territory).

Experiment 1. Hedonic hot spot for taste reactivity

Localized shell site for DAMGO: enhancement of sucrose "liking"

Microinjections of the DAMGO caused increases in the number of positive hedonic "liking" reactions elicited by infusions of sucrose solution into rats' mouths, but only in a particular rostral region of medial shell (Fig. 2A, $B$, supplemental Fig. S1, available at www.jneurosci.org as supplemental material). This hedonic subregion or "liking" hot spot of accumbens shell was located in the rostral half of the medial shell and slightly dorsal within it, just anterior to the caudal edge of the islands of Calleja but posterior to the caudal edge of the dorsal tenia tecta and the lateral septum (lying between AP +2.0 and +1.0 $\mathrm{mm}$ from bregma) and at or dorsal to the level of the anterior commissure (lying between DV -6.4 and $-7.5 \mathrm{~mm}$ from skull surface) (Paxinos and Watson, 1996). The size of this "positive hedonic hot spot" where DAMGO amplified sucrose "liking" reactions was $1 \mathrm{~mm}^{3}$ in volume for the lower $0.01 \mu \mathrm{g}$ dose $\left[1.00 \mathrm{~mm}^{3}\right.$ volume; $\sim 1$ $\mathrm{mm}$ AP $\times$ mm DV $\left(1.12 \mathrm{~mm}^{2}\right.$ in sagittal section area) $\times 0.9 \mathrm{~mm}$ ML thickness of medial shell] and just over $1 \mathrm{~mm}^{3}$ for the higher $0.1 \mu \mathrm{g}$ dose $\left(1.15 \mathrm{~mm}^{3}\right.$ volume; $1.28 \mathrm{~mm}^{2}$ sagittal area).

Within this $\sim 1 \mathrm{~mm}^{3}$ hot spot in the rostrodorsal region of the medial shell, both doses of DAMGO enabled sucrose taste to elicit over double the control number of positive hedonic reactions compared with after vehicle microinjections [two-way ANOVA (site-by-drug); $F_{(1,199)}=13.64, p<0.001$, main effect of site; $F_{(1,199)}=8.23, p<0.001$, main effect of drug]. The lower $0.01 \mu \mathrm{g}$ dose of DAMGO in this spot increased positive hedonic reactions to sucrose by $200 \%$ above vehicle, and the higher $0.1 \mu \mathrm{g}$ dose tripled them by 325\% (both $p<0.05$, Tukey's test). The hedonic hot spot overlaps with the so-called caudal shell region we previously reported to support increases in positive hedonic taste reactions$$
\leftarrow
$$

supplemental Fig. S1 for 3-D horizontal maps of hedonic hot spot; insets show examples of hedonic "liking" reactions to sucrose by rat and homologous equivalent expressions by human infant and disliking reactions to quinine; see supplemental Movie 1 for examples of positive hedonic reactions to sucrose taste and negative aversive reactions to quinine taste). Error bars represent SEM. LSI, Lateral septum intermediate; LSV, lateral septum ventral; VP, ventral pallidum. 
and food intake after morphine microinjections (Peciña and Berridge, 2000), but we note that our previous study was restricted to sites in the rostral half of the shell and did not explore the caudal half of medial shell posterior to islands of Calleja. Our present more extensive mapping of medial shell indicates that opioid substrates for generating increased "liking" reactions to positive hedonic impact of sweetness are actually contained within the rostral half [albeit concentrated caudally (and dorsally) within it]: essentially the rostral dorsal quarter of medial shell (and to be most precise, the midrostral dorsal portion of medial shell, or the posterior one-half of the rostral dorsal quarter of the medial shell) (see Figs. 2, 4, supplemental Figs. S1, S2, S3, available at www. jneurosci.org as supplemental material).

In contrast, DAMGO microinjections at all other sites in the medial shell failed to increase positive hedonic reactions (despite stimulating increase food intake, discussed below). For example, no significant changes in "liking" reactions were produced at all in any grid square in the entire ventral half of the accumbens medial shell (DV, 7.4-8.6 mm; one-way ANOVA; $F_{(1,101)}=0.23 ; p=0.35$, NS). The lack of DAMGO effect at those sites differed significantly from the DAMGO increase in hedonic reactions of the dorsal hot spot (site interaction; $\left.F_{(1,199)}=13.64 ; p<0.001\right)$.

Rostral versus caudal differences were even more dramatic, because DAMGO microinjections in a small zone in the caudal half of the medial shell $(\sim 1 \mathrm{~mm}$ caudal to the "liking" hot spot) actually appeared to suppress sucrose-elicited "liking" reactions below vehicle control levels $\left(F_{(1,116)}\right.$ $=8.45 ; p<0.05$ ) (Fig. 2). We therefore considered this caudal shell zone a potential opioid hedonic "cold spot" where opioid activation might actually inhibit hedonic impact (blue in Fig 2 and supplemental Fig. S1, available at www.jneurosci.org as supplemental material) (drug-by-site; $\left.F_{(1,116)}=9.25 ; p<0.05\right)$. When doses were considered separately, the highest $0.1 \mu \mathrm{g}$ DAMGO dose by itself produced significant suppression of sucrose-elicited reactions at this caudal site $\left(F_{(2,17)}=8.73 ; p<0.05\right)$ (Fig. $\left.2 B\right)$, and the lower 0.01 dose by itself produced a marginal trend toward suppression $\left(F_{(2,17)}=\right.$ $4.32 ; p=0.12$ ) (Fig. 2A). DAMGO also failed to increase hedonic reactions in the farthest rostral strip, constituting the most rostral $20 \%$ of medial shell (AP, $2.0-2.5 \mathrm{~mm}$ from bregma; one-way ANOVA; $F_{(1,23)}=0.56 ; p=0.35$, NS) (Fig. 3). Previous studies have similarly found that manipulation effects in this farthest extreme rostral strip differ from those in the rest of the rostral half of medial shell (Reynolds and Berridge, 2002).

DAMGO suppression of aversive quinine "disliking" reactions DAMGO microinjections in the medial shell also suppressed aversive reactions elicited by quinine taste overall (one-way

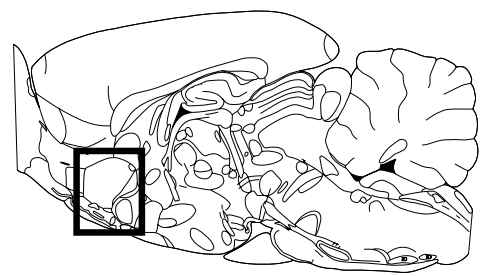

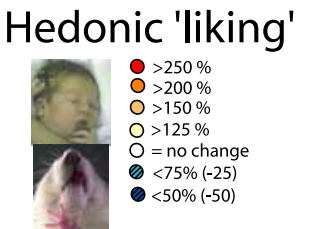
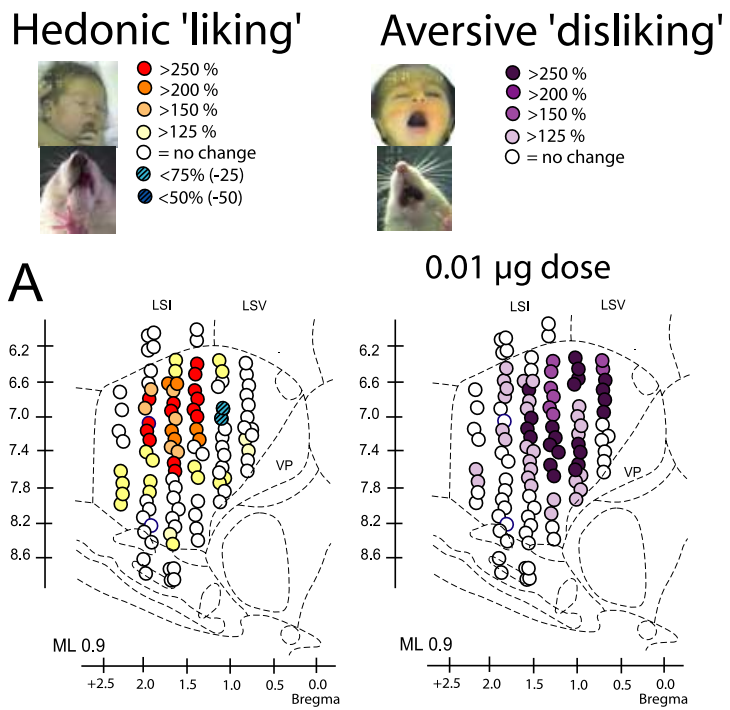

$0.01 \mu \mathrm{g}$ dose

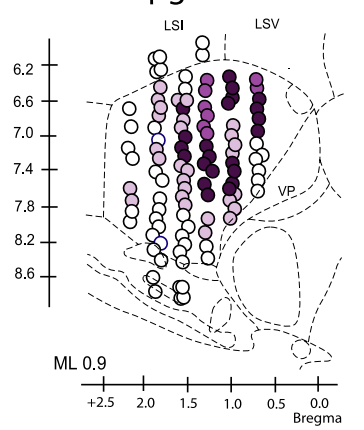

$0.1 \mu \mathrm{g}$ dose

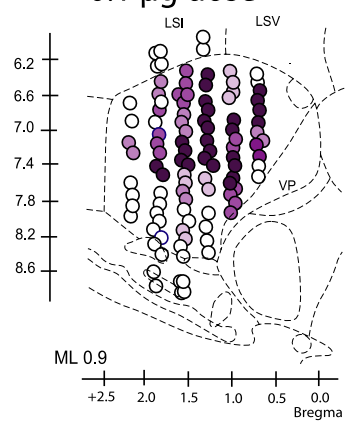

Food intake
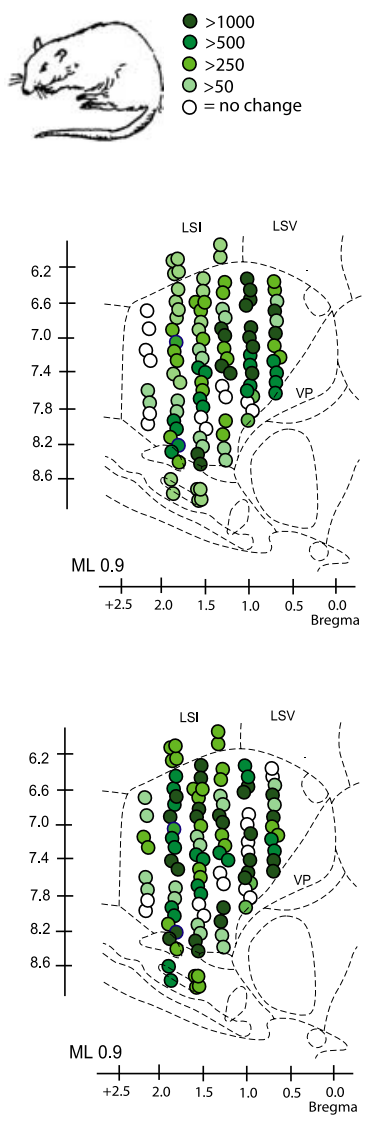

Figure 3. Pinpoint map of microinjection centers and their effects. Increases in sucrose "liking" reactions, suppression of quinine "disliking" reactions, and stimulation of food intake are color-coded as in Figure 2. The pinpoint maps do not show the degree of diffusion from site centers but are still helpful to show functional differences among individual sites (such as the relative lack of effect of site centers in the most extreme far rostral $10-20 \%$ column of medial shell, as well as some localization effects shown in Fig. 2).

ANOVA; $F_{(2,127)}=8.26 ; p<0.05$ ) (Fig. $2 C, D$ ). Suppression of aversive "disliking" reactions was site specific and interestingly was also strongest in the caudal half of medial shell $(>250 \%$; $\left.F_{(1,137)}=5.58 ; p<0.05\right)$. The overlap of strongest suppressed quinine aversion with the hedonic cold spot above suggests that $\mu$-opioid activation in this caudal zone, which was $\sim 2 \mathrm{~mm}^{3}$ in volume $\left(2.01 \mathrm{~mm}^{3}\right)$, may suppress all affective reactions (both positive and negative), whereas the rostral hedonic hot spot more specifically amplifies only positive "liking" reactions to sweetness hedonic impact. Only at the intersecting edge of hot spot and cold spot did DAMGO cause increases in positive hedonic sucrose "liking" and decreases in quinine "disliking" or simultaneous symmetrical shift in positive and negative affective reactions.

The "affective cold spot" for suppressing positive/negative affective reactions appeared to be posterior to the major island of the islands of Calleja and extended posteriorly to the caudal edge of the accumbens shell (AP, between +1.5 and $+0.5 \mathrm{~mm}$ from bregma) (Paxinos and Watson, 1996). When doses were consid- 


\section{A Hedonic vs intake contrast}

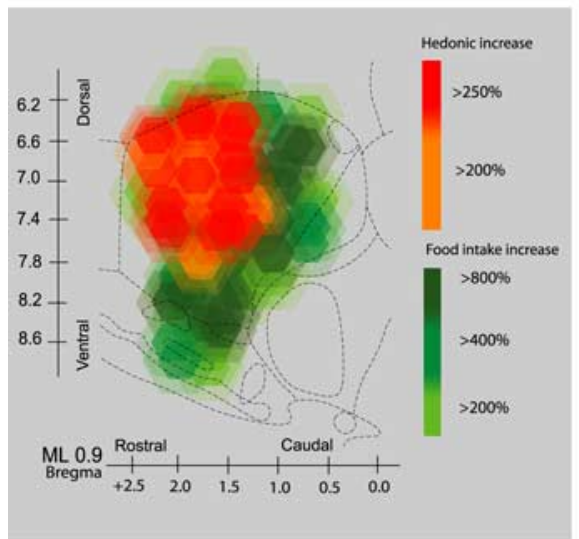

B Hedonic vs aversive contrast

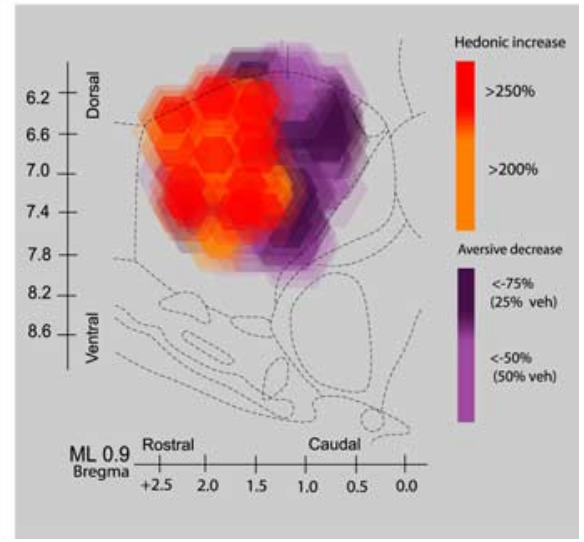

Figure 4. Contrast maps for opioid hedonic hot spot versus distributed food intake $(\boldsymbol{A})$ and affective cold spot $(\boldsymbol{B})$. $\boldsymbol{A}$, Combined summary map of DAMGO ( 0.01 and $0.1 \mu \mathrm{g}$ doses) hedonic enhancement effects on positive hedonic "liking" reactions to sucrose (shown in red/orange/yellow) versus stimulation of food intake (green). Each rat's dose effects were combined to produce a single hedonic impact increase score for its site and a single food intake increase score. A mapping threshold was set at $>200 \%$ increase over vehicle (veh) control levels for both hedonic and intake effects. Thus, color reflects only sites where DAMGO caused average doubling or more of hedonic reactions or of food intake. Hedonic effects are mapped on top of intake effects. Red/yellow hedonic hot spot shows where DAMG0 caused both increased hedonic "liking" reactions and increased food intake. Blue/green shows areas where DAMGO increased food intake without increasing hedonic "liking" reactions to taste. $\boldsymbol{B}$, Combined summary map for hedonic hot spot (red/yellow) versus affective cold spot where DAMGO suppressed aversion (purple). Mapping threshold for hedonic hot spot again required DAMGO at a site to double the number of positive reactions elicited by sucrose taste. Threshold for mapping cold spot suppression required DAMGO at that site to cut in half the number of aversive reactions elicited by quinine taste (both 0.01 and $0.1 \mu \mathrm{g}$ dose effects combined for each rat). Hedonic enhancement was mapped on top of aversive suppression (i.e., aversive suppression extends under red/yellow zone).

ered separately, both 0.01 and $0.01 \mu \mathrm{g}$ doses of DAMGO each suppressed aversive reactions in this caudal site $(p<0.05$; Tukey's test).

\section{Control sites}

At our control sites outside the medial shell, either dorsal or ventral to the nucleus accumbens, DAMGO failed to increase or decrease taste-elicited hedonic or aversive reactions that sites. For example, no changes in sucrose-elicited "liking" reactions were produced by DAMGO in either the lateral septum (dorsal to the accumbens medial shell $)\left(F_{(2,11)}=2.51 ; p=\right.$ NS) or in olfactory tubercle sites ventral to the medial shell $\left(F_{(2,5)}=1.474 ; p=\mathrm{NS}\right)$ or in other regions of the medial shell itself beyond those described above (Figs. 2A, B, 3). Additional work remains to be done to map opioid hedonic impact effects (or lack thereof) in other nucleus accumbens regions such as the ventrolateral shell and core, as well as adjacent brain structures. For now, our results indicate that $\mu$-opioid modulation of positive "liking" reactions to sweet reward is surprisingly localized to a specific rostral positive hedonic hot spot in medial shell and that there may be a caudal shell cold spot for general affective inhibition by $\mu$-opioid action.

\section{Experiment 2: DAMGO increases food intake throughout the medial accumbens shell}

The duration of eating behavior and food intake was increased by DAMGO microinjections at virtually all sites throughout the entire medial shell, especially at the highest dose [two-way ANOVA (site-by-drug); $F_{(1,137)}=10.24 ; p<0.001$; main effect of drug] (Fig. 2E,F). DAMGO nearly always doubled duration of eating, and often quadrupled it or more, at nearly all microinjection sites. Despite the rostral hot spot for hedonic impact described above, caudal sites appeared to be at least as effective for stimulating food intake, and caudal sites may even have produced greater increases than rostral sites, especially at the low dose [two-way ANOVA (site-by-drug); $F_{(1,137)}=8.26 ; p=0.05$ ]. At the lowest $0.01 \mu \mathrm{g}$ DAMGO dose, the caudal half of the shell supported higher increases in eating duration than the rostral site $\left(F_{(1,137)}=8.26 ; p=0.05\right)$ (Fig. $2 E)$. However, the higher 0.1 $\mu \mathrm{g}$ DAMGO dose elicited equally robust eating from both rostral and caudal shell ( $>800 \%$ increase at several sites, including hedonic hot spot and aversive cold spot described above) (Fig. 2 F), so that rostral and caudal halves no longer differed significantly. This pattern of results suggests a potential rostrocaudal gradient of threshold sensitivity to DAMGO effects on food intake: highly sensitive caudal sites are activated by low DAMGO doses, but more rostral sites require higher DAMGO stimulation to be fully recruited. An alternative explanation is that higher doses diffuse from rostral sites into caudal zones, but the size of Fos plumes suggest that the range of effective diffusion did not much exceed $0.5 \mathrm{~mm}$, even for the higher dose. It therefore is likely that these maps accurately reflect localization of function with under a $0.5 \mathrm{~mm}$ margin of error. It is perhaps important to note that even our high dose of DAMGO was lower than doses used by most previous accumbens microinjection studies of food intake, in which diffusion might conceivably play greater roles.

Thus, nearly all regions of the medial shell appear able to cause dramatic increases in food intake in response to sufficient $\mu$-opioid stimulation, consistent with previous reports (Ragnauth et al., 2000; Zhang and Kelley, 2000; Kim et al., 2004). The only medial shell region where DAMGO failed to increase eating was the farthest rostral $0.5 \mathrm{~mm}$ strip of medial shell, where neither dose evoked increased food intake above vehicle levels (anterior to +2.0 bregma) (Figs. 2E, F, 3). Again, this farthest rostral strip has been reported also to differ from the rest of medial shell in behavioral effects produced by microinjections of muscimol, a GABA agonist (Reynolds and Berridge, 2002), supporting the notion that the far rostral $10-20 \%$ of the medial shell may respond differently from the rest of the medial shell to neurochemical manipulation. In summary, DAMGO elicits robust eating behavior at virtually all sites throughout the entire medial shell, except in the far rostral strip. At lower doses caudal sites may have a slight advantage for stimulating food intake.

\section{Discussion}

We found that $\mu$-opioid activation by DAMGO within a restricted $1 \mathrm{~mm}^{3}$ site in the rostrodorsal medial shell amplified the hedonic impact of sweetness as reflected by more positive "liking" reactions elicited by sucrose in a taste reactivity analysis (Fig. 4). Our findings confirm that opioid circuits in the nucleus accumbens cause amplified positive hedonic impact of natural rewards and help pinpoint their precise location. In contrast, $\mu$-opioid substrates of DAMGO-increased food intake were much more distributed anatomically, including nearly every site tested throughout the entire medial shell (Fig. 4A, supplemental Fig. S2, available at www.jneurosci.org as supplemental mate- 
rial). Food intake was increased even in the affective cold spot where DAMGO suppressed both sucrose "liking" and quinine "disliking" affective reactions.

\section{Hedonic hot spot for "liking" enhancement}

The hedonic hot spot for DAMGO enhancement of sucroseelicited "liking" reactions was localized in the rostral and dorsal quarter of medial shell (Fig. 4). This falls within the same rostral half where appetitive food intake, conditioned place preference effects are best produced by microinjections of AMPA/kainate glutamate receptor antagonist $(\mathrm{DNQX})$ or by $\mathrm{GABA}_{\mathrm{A}}$ agonist (muscimol) (Reynolds and Berridge, 2001, 2002, 2003), although our opioid hot spot here was even more anatomically restricted.

DAMGO in this rostral and dorsal hedonic hot spot caused sucrose to elicit doubled or quadrupled numbers of positive "liking" reactions to sucrose and simultaneously decreased aversive reactions to quinine to under $25 \%$ of control levels. That shift toward hedonic enhancement is exactly what should be expected if opioid circuits increase the positive hedonic impact of sensory pleasure, while reducing the negative aversive impact of sensory displeasure. However, the apparent anatomical separation of the hedonic hot spot (where DAMGO selectively enhanced positive "liking" reactions) and the affective cold spot (where DAMGO suppressed reactions indicative of both positive hedonic impact and negative "disliking" or aversion) suggests these two aspects of hedonic amelioration by opioid circuits may be somewhat segregated (Fig. $4 B$, supplemental Fig. S3, available at www.jneurosci. org as supplemental material). A localized rostral bias in the medial shell may apply to opioid magnification of positive hedonic impact, whereas a caudal bias may apply to opioid suppression of affective reactions (to suppress both positive hedonic and negative aversive reactions). These two affective sites overlap only at their intersecting edge. At that intersection, functional enhancement of positive "liking" reactions wins over suppression, so that in that zone positive reactions to sucrose are increased while negative reactions to quinine remain decreased.

Some grounds for confidence in this localization comes from the observations of Fos plumes indicating that effective activation spread was limited to a $0.2-0.5 \mathrm{~mm}$ radius from microinjection centers, suggesting that hedonic impact effects were not mediated by diffusion of DAMGO to act elsewhere. Control sites in structures outside the nucleus accumbens such as the lateral septum and olfactory tubercle were also ineffective at modulating "liking"/ "disliking" reactions to sweet or bitter tastes here, although such sites are linked to other appetitive effects of drugs (Ikemoto, 2003). Also, most of the medial shell itself was silent for hedonic modulation outside the hot spots and cold spots.

\section{Hedonic function maps: coding and causal roles}

What is the role of the opioid hot spot in mediating hedonic impact of rewards? First, regarding types of reward, it is important to note we mapped opioid enhancement of a natural reward (sweet taste), rather than hedonic impact of the opioid drug itself. It would not be unreasonable to expect that both share similar hedonic substrates, because brain reward circuits evolved to process natural rewards, and drugs of abuse must modulate those natural reward circuits. Still, the precise relationship of drug reward to natural hedonic localization remains an open empirical question.

Second, even within the domain of natural reward, it is helpful to distinguish between causation versus coding of hedonic impact. For example, we cannot predict whether neuroimaging, electrophysiological, or neurochemical release studies would find coding of hedonic impact in medial shell to be as anatomically restricted as our causation study. In other words, hedonic coding may turn out to be more anatomically distributed than hedonic causation. Finally, within types of neural causation itself, there may be differences between being a sufficient cause versus a necessary cause of hedonic impact. Our results indicate $\mu$-opioid activation in the shell hedonic hot spot is a sufficient cause to amplify positive hedonic impact, at least in the sense of causing increased hedonic "liking" reactions to sweet sensation when other brain conditions remain normal. But that need not imply that losses of normal hedonic reactions to sweetness would necessarily result from lesions of the medial shell or that the hot spot is a necessary cause for normal hedonic impact. In other words, circuits for different types of hedonic causation might be differentially distributed in the brain. The substrates necessary to mediate normal positive hedonic reactions to sweetness may survive damage to certain components, such as circuits in the hot spot of medial shell, where opioid activation otherwise causes increased enhancement of hedonic reactions to natural reward impact.

\section{Entire medial shell mediates opioid increase of food intake}

DAMGO throughout the entire shell increased food intake, confirming previous reports of wide nucleus accumbens distribution (Ragnauth et al., 2000; Zhang and Kelley, 2000; Kim et al., 2004). Food intake was more than quadrupled even at the caudal affective cold spot where DAMGO suppressed all affective reactions to tastes (Fig. 4B). If anything, caudal microinjections were more able than rostral microinjections to increase intake at the low 0.01 $\mu \mathrm{g}$ dose of DAMGO. Observation of increased eating without enhanced hedonic impact appears somewhat similar to dissociations of food "wanting" from "liking" produced by other manipulations of mesolimbic function (Berridge and Valenstein, 1991; Reynolds and Berridge, 2002; Wyvell and Berridge, 2000; Peciña et al., 2003).

\section{Neuroanatomical compartmentalization in medial shell}

The medial shell of the nucleus accumbens contains subregional differences in neurotransmitter and receptor distribution and anatomical connectivity patterns, which might be relevant to our localization here of an opioid hedonic hot spot. For example, $\mu$-opioid receptors are particularly dense in the rostral and dorsal medial shell containing our hedonic hot spot, compared with other regions (Tempel and Zukin, 1987).

Regarding rostrocaudal differences in anatomical connectivity, the rostromedial shell receives denser excitatory projections than the caudal shell from dorsal intermediate subiculum, entorhinal cortex, and rostral prelimbic area. By comparison, the caudal shell receives greater inputs from ventral subiculum, septohippocampal area, basal amygdaloid complex, caudal prelimbic area, and brainstem norepinephrine projections (Phillipson and Griffiths, 1985; Groenewegen et al., 1987; Berridge et al., 1997). Furthermore, convergence onto single accumbens neurons from hippocampal and amygdaloid inputs occurs more in the caudal and intermedial shell than the rostral shell (Mulder et al., 1998).

Regarding dorsoventral differences, the dorsal half of the medial shell receives more fibers than the ventral shell from the parvicellular basal nucleus, medial amygdala nucleus, caudal periamygdaloid cortex, and basal parvicellular amygdaloid nucleus (Alheid and Heimer, 1988; Brog et al., 1993; Fudge et al., 2002). Furthermore, only the dorsal shell receives converging input from both the medial amygdala nucleus and central and periamygdaloid regions, at least in primates (Fudge et al., 2002). The ventral shell receives more inputs from accessory basal and 
magnocellular basal nuclei (Wright et al., 1996). In efferent projections, the dorsal shell sends more outputs to the medioventral tegmental area (Voorn et al., 1986; Berendse et al., 1992), whereas the ventral shell sends more to the lateroventral tegmental area (Berendse et al., 1992).

Connectivity could conceivably relate hedonic localization in the medial shell to related hedonic hot spots in other limbic brain structures. For example, in the ventral pallidum, DAMGO microinjections in the posterior subregion cause increases in hedonic "liking" reactions to sucrose taste similar to the hedonic hot spot here, whereas the same opioid microinjections in anterior subregions of ventral pallidum only inhibit sucrose "liking" reactions (Smith and Berridge, 2005). In the ventral pallidum, however, opioid-induced enhancement of sucrose hedonic impact seems more tightly linked to opioid-induced stimulation of food intake than here in medial shell, and it is not yet known whether opioid hedonic sites in these two brain structures have any special anatomical connection between them. Finally, it seems noteworthy that some patterns of connectivity differences within the nucleus accumbens medial shell are organized in localized clusters, which might be important to explaining why we find opioid substrates for enhancing hedonic impact are packed into a single cubic millimeter site (Heimer et al., 1997). Although their role in explaining our functional localization of hedonic impact remains unknown, these patterns of anatomical differences within medial shell at least provide candidates for future study.

\section{Conclusion}

Our mapping study of $\mu$-opioid substrates for enhancing hedonic "liking" reactions to sweetness pleasure and stimulating food intake reveals an anatomically distinct hedonic hot spot, $\sim 1$ $\mathrm{mm}^{3}$ in unilateral size, localized within the rostral and dorsal quarter of the medial shell of nucleus accumbens. In contrast, $\mu$-opioid substrates that stimulate food intake and eating behavior are more widely anatomically distributed throughout the medial accumbens shell. These findings provide insight into the identity of nucleus accumbens mechanisms that mediate sensory pleasure and suggest that opioid circuits in much of the medial shell outside the "liking" hot spot may stimulate food intake by a neurobehavioral mechanism that is separable from hedonic impact.

\section{References}

Alheid GF, Heimer L (1988) New perspectives in basal forebrain organization of special relevance for neuropsychiatric disorders: the striatopallidal, amygdaloid, and corticopetal components of substantia innominata. Neuroscience 27:1-39.

Berendse HW, Galis-de Graaf Y, Groenewegen HJ (1992) Topographical organization and relationship with ventral striatal compartments of prefrontal corticostriatal projections in the rat. J Comp Neurol 316:314-347.

Berridge CW, Stratford TL, Foote SL, Kelley AE (1997) Distribution of dopamine beta-hydroxylase-like immunoreactive fibers within the shell subregion of the nucleus accumbens. Synapse 27:230-241.

Berridge KC (1996) Food reward: brain substrates of wanting and liking. Neurosci Biobehav Rev 20:1-25.

Berridge KC (2000) Measuring hedonic impact in animals and infants: microstructure of affective taste reactivity patterns. Neurosci Biobehav Rev 24:173-198.

Berridge KC (2003) Pleasures of the brain. Brain Cogn 52:106-128.

Berridge KC, Valenstein ES (1991) What psychological process mediates feeding evoked by electrical stimulation of the lateral hypothalamus? Behav Neurosci 105:3-14.

Bodnar RJ, Glass MJ, Ragnauth A, Cooper ML (1995) General, mu and kappa-opioid antagonists in the nucleus-accumbens alter food-intake under deprivation, glucoprivic and palatable conditions. Brain Res 700:205-212.
Bodnar RJ, Lamonte N, Israel Y, Kandov Y, Ackerman TF, Khaimova E (2005) Reciprocal opioid-opioid interactions between the ventral tegmental area and nucleus accumbens regions in mediating mu agonistinduced feeding in rats. Peptides 26:621-629.

Brog JS, Salyapongse A, Deutch AY, Zahm DS (1993) The patterns of afferent innervation of the core and shell in the "accumbens" part of the rat ventral striatum: immunohistochemical detection of retrogradely transported fluoro-gold. J Comp Neurol 338:255-278.

Cooper SJ, Higgs S (1994) Neuropharmacology of appetite and taste preferences. In: Appetite: neural and behavioural bases (Legg CR, Booth DA, eds), pp 212-242. New York: Oxford UP.

Doyle TG, Berridge KC, Gosnell BA (1993) Morphine enhances hedonic taste palatability in rats. Pharmacol Biochem Behav 46:745-749.

Echo JA, Lamonte N, Ackerman TF, Bodnar RJ (2002) Alterations in food intake elicited by GABA and opioid agonists and antagonists administered into the ventral tegmental area region of rats. Physiol Behav 76:107-116.

Evans KR, Vaccarino FJ (1990) Amphetamine- and morphine-induced feeding: evidence for involvement of reward mechanisms. Neurosci Biobehav Rev 14:9-22.

Fudge JL, Kunishio K, Walsh P, Richard C, Haber SN (2002) Amygdaloid projections to ventromedial striatal subterritories in the primate. Neuroscience 110:257-275.

Grill HJ, Berridge KC (1985) Taste reactivity as a measure of the neural control of palatability. In: Progress in psychobiology and physiological psychology (Sprague JM, Epstein AN, eds), pp 1-61. Orlando, FL: Academic.

Grill HJ, Norgren R (1978) The taste reactivity test. I. Mimetic responses to gustatory stimuli in neurologically normal rats. Brain Res 143:263-279.

Groenewegen HJ, Vermeulen-Van der Zee E, te Kortschot A, Witter MP (1987) Organization of the projections from the subiculum to the ventral striatum in the rat. A study using anterograde transport of Phaseolus vulgaris leucoagglutinin. Neuroscience 23:103-120.

Heimer L, Alheid GF, de Olmos JS, Groenewegen HJ, Haber SN, Harlan RE, Zahm DS (1997) The accumbens: beyond the core-shell dichotomy. J Neuropsychiatry Clin Neurosci 9:354-381.

Ikemoto S (2003) Involvement of the olfactory tubercle in cocaine reward: intracranial self-administration studies. J Neurosci 23:9305-9311.

Kalivas PW, Volkow ND (2005) The neural basis of addiction: a pathology of motivation and choice. Am J Psychiatry 162:1403-1413.

Kelley AE (2004) Ventral striatal control of appetitive motivation: role in ingestive behavior and reward-related learning. Neurosci Biobehav Rev 27:765-776.

Kelley AE, Bless EP, Swanson CJ (1996) Investigation of the effects of opiate antagonists infused into the nucleus accumbens on feeding and sucrose drinking in rats. J Pharmacol Exp Ther 278:1499-1507.

Kelley AE, Bakshi VP, Haber SN, Steininger TL, Will MJ, Zhang M (2002) Opioid modulation of taste hedonics within the ventral striatum. Physiol Behav 76:365-377.

Kim EM, Quinn JG, Levine AS, O'Hare E (2004) A bi-directional muopioid-opioid connection between the nucleus of the accumbens shell and the central nucleus of the amygdala in the rat. Brain Res 1029:135-139.

Koob GF (2003) Alcoholism: allostasis and beyond. Alcohol Clin Exp Res 27:232-243.

Levine AS, Billington CJ (1997) Why do we eat? A neural systems approach. Annu Rev Nutr 17:597-619.

Levine AS, Olszewski PK, Mullett MA, Pomonis JD, Grace MK, Kotz CM, Billington CJ (2004) Intra-amygdalar injection of DAMGO: effects on c-Fos levels in brain sites associated with feeding behavior. Brain Res 1015:9-14.

Majeed NH, Przewlocka B, Wedzony K, Przewlocki R (1986) Stimulation of food intake following opioid microinjection into the nucleus accumbens septi in rats. Peptides 7:711-716.

Mucha RF, Iversen SD (1986) Increased food intake after opioid microinjections into nucleus accumbens and ventral tegmental area of rat. Brain Res 397:214-224.

Mulder AB, Hodenpijl MG, Lopes da Silva FH (1998) Electrophysiology of the hippocampal and amygdaloid projections to the nucleus accumbens of the rat: convergence, segregation, and interaction of inputs. J Neurosci 18:5095-5102.

Muller R, Bravo R, Burckhardt J, Curran T (1984) Induction of c-fos gene 
and protein by growth factors precedes activation of c-myc. Nature 312:716-720

Parker LA, Maier S, Rennie M, Crebolder J (1992) Morphine- and naltrexone-induced modification of palatability: analysis by the taste reactivity test. Behav Neurosci 106:999-1010.

Paxinos G, Watson C (1996) The rat brain in stereotaxic coordinates. New York: Academic.

Peciña S, Berridge KC (1995) Central enhancement of taste pleasure by intraventricular morphine. Neurobiology 3:269-280.

Peciña S, Berridge KC (2000) Opioid eating site in accumbens shell mediates food intake and hedonic 'liking': map based on microinjection Fos plumes. Brain Res 863:71-86.

Peciña S, Cagniard B, Berridge KC, Aldridge JW, Zhuang X (2003) Hyperdopaminergic mutant mice have higher "wanting" but not "liking" for sweet rewards. J Neurosci 23:9395-9402.

Phillipson OT, Griffiths AC (1985) The topographic order of inputs to nucleus accumbens in the rat. Neuroscience 16:275-296.

Ragnauth A, Moroz M, Bodnar RJ (2000) Multiple opioid receptors mediate feeding elicited by mu and delta opioid receptor subtype agonists in the nucleus accumbens shell in rats. Brain Res 876:76-87.

Reynolds SM, Berridge KC (2001) Fear and feeding in the nucleus accumbens shell: rostrocaudal segregation of GABA-elicited defensive behavior versus eating behavior. J Neurosci 21:3261-3270.

Reynolds SM, Berridge KC (2002) Positive and negative motivation in nucleus accumbens shell: bivalent rostrocaudal gradients for GABA-elicited eating, taste "liking"/“disliking" reactions, place preference/avoidance, and fear. J Neurosci 22:7308-7320.

Reynolds SM, Berridge KC (2003) Glutamate motivational ensembles in nucleus accumbens: rostrocaudal shell gradients of fear and feeding in the rat. Eur J Neurosci 17:2187-2200.

Rideout HJ, Parker LA (1996) Morphine enhancement of sucrose palatabil- ity: analysis by the taste reactivity test. Pharmacol Biochem Behav 53:731-734.

Robbins TW, Everitt BJ (2002) Limbic-striatal memory systems and drug addiction. Neurobiol Learn Mem 78:625-636.

Smith KS, Berridge KC (2005) The ventral pallidum and hedonic reward: neurochemical maps of sucrose "liking" and food intake. J Neurosci 25:8637-8649.

Tempel A, Zukin RS (1987) Neuroanatomical patterns of the mu, delta, and kappa opioid receptors of rat brain as determined by quantitative in vitro autoradiography. Proc Natl Acad Sci USA 84:4308-4312.

Voorn P, Jorritsma-Byham B, Van Dijk C, Buijs RM (1986) The dopaminergic innervation of the ventral striatum in the rat: a light- and electronmicroscopical study with antibodies against dopamine. J Comp Neurol 251:84-99.

Will MJ, Franzblau EB, Kelley AE (2004) The amygdala is critical for opioidmediated binge eating of fat. NeuroReport 15:1857-1860.

Wright CI, Beijer AV, Groenewegen HJ (1996) Basal amygdaloid complex afferents to the rat nucleus accumbens are compartmentally organized. J Neurosci 16:1877-1893.

Wyvell CL, Berridge KC (2000) Intra-accumbens amphetamine increases the conditioned incentive salience of sucrose reward: enhancement of reward "wanting" without enhanced "liking" or response reinforcement. J Neurosci 20:8122-8130.

Zhang M, Kelley AE (1997) Opiate agonists microinjected into the nucleus accumbens enhance sucrose drinking in rats. Psychopharmacology $132: 350-360$

Zhang M, Kelley AE (2000) Enhanced intake of high-fat food following striatal mu-opioid stimulation: microinjection mapping and fos expression. Neuroscience 99:267-277.

Zhang M, Gosnell BA, Kelley AE (1998) Intake of high-fat food is selectively enhanced by mu opioid receptor stimulation within the nucleus accumbens. J Pharmacol Exp Ther 285:908-914. 